\title{
The treatment of small delay case in linear multistep method
}

\begin{abstract}
This article presents a technique on how to deal with the presence of small delay case in delay differential equations. This case has been classified as one of the difficulties in the numerical solution where it is due to the delay value that is smaller than the current step size and there is no current approximate solution that can be used in the interpolating of delay value. In this technique we will highlight on extrapolation and interpolation approaches depending on the existence of delay value in the grid point. The computational method for determining the sequence of $\{\mathrm{yn}\}$ by using the linear multistep method is investigated with the implementation of variable step size. The numerical experiments were tested on the several problems and results were compared with other approaches from those of known solvers in treating the small delay case.
\end{abstract}

Keyword: Small delay; Delay differential equations; Linear multistep method; Variable step size 